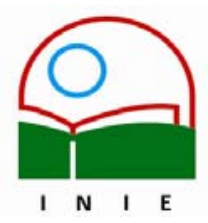

Universidad de Costa Rica

Facultad de Educación

Instituto de Investigación en Educación

ACTUALIDADES INVESTIGATIVAS EN EDUCACION

\title{
UNA REVISIÓN CRÍTICA DEL CONCEPTO DE CREATIVIDAD
}

\author{
Yamileth Chacón Araya ${ }^{1}$
}

\begin{abstract}
Resumen: El presente artículo tiene como objetivos resumir el estado actual del conocimiento en el campo de la creatividad y presentar una nueva perspectiva de lo planteado por diferentes autores sobre este tema particular. $A$ la vez, se busca establecer un acercamiento a la utilización de este concepto dentro del campo educativo, principalmente el universitario, y su relación específica con las ciencias del movimiento humano.

La creatividad toma una importancia primordial dentro del desarrollo humano de acuerdo con los diferentes enfoques de investigación y los diversos modelos conceptuales que se han propuesto a través de los años. Esta conclusión se basa en los resultados de las innumerables investigaciones sobre creatividad. Por esa razón, se busca integrar los trabajos publicados por algunos autores con respecto a este complejo constructo.

En primera instancia, se reúnen y se discuten algunos conceptos que tienen relación con esta visión.. También se discuten las teorías sobre la creatividad y luego se plantean y se describen algunas características que parecen comunes en las personas creativas. Además, se detallan aspectos básicos sobre la importancia de la creatividad en la educación y en las ciencias del movimiento humano. Finalmente, se proponen algunas líneas de investigación, especialmente en el área de las ciencias del movimiento humano.
\end{abstract}

\section{Palabras claves: CREATIVIDAD/ EDUCACIÓN/ CIENCIAS DEL MOVIMIENTO HUMANO/}

\begin{abstract}
The purpose of this paper is to summarize the state of the knowledge in creativity research and to present a new perspective to what has been published before. A secondary aim is to look for a different approach to use this construct in the educational area, mainly at the college level, and how it relates to the human movement sciences.

Based on different research and conceptual approaches carried out throughout the years, it has been proposed that the creativity is a fundamental piece of the human development process. This conclusion is based on the results of several investigations on creativity; therefore, it seems important to integrate the knowledge from the works published by some authors in regard to this complex construct.

First, in order to better understand the problem, a series of concepts related to creativity are discussed. Secondly, different theories on creativity are presented; then, common characteristics of creative people are described. Key factors are detailed on the importance of creativity in education and especially within the human movement sciences. Finally, potential research on creativity within the human movement sciences is suggested.
\end{abstract}

Key words: CREATIVITY/ EDUCATION/ HUMAN MOVEMENT SCIENCES/

\begin{abstract}
${ }^{1}$ Maestría en Psicopedagogía en la Universidad Nacional Estatal a Distancia, el énfasis de la maestría fue en el área de la creatividad en los estudiantes universitarios. Bachillerato y Licenciatura en la Enseñanza de la Educación Física de la Universidad de Costa Rica. Imparte cursos en la Escuela de Educación Física y Deportes de la Universidad de Costa Rica desde 1991. Coordinadora de Vida Estudiantil de la Unidad Académica y coordinadora de la comisión organizadora del Simposio Internacional en Ciencias del Deporte, el Ejercicio y la Salud y del Congreso Internacional de Ciencias Aplicadas al Fútbol. Ha sido coordinadora de los cursos de verano, en el área de extensión docente.
\end{abstract}

Correo electrónico: ychacon@cariari.ucr.ac.cr

Artículo recibido: 6 de mayo, 2005

Aprobado: 20 de junio, 2005 


\section{Introducción}

La palabra creatividad aparece continuamente en los medios de comunicación colectiva y parece ser una herramienta muy importante en diferentes campos de trabajo; entre otras cosas, para la búsqueda del personal idóneo en las empresas. En otros ámbitos, la creatividad se relaciona fuertemente con la expresión del arte, la música, la ciencia, y se ha mencionado que puede ser una herramienta indispensable para renovar la educación y cambiar el futuro.

En la actualidad, se considera importante estimular la creatividad, utilizando estrategias que se apeguen a las disciplinas que sirven de guía a las diversas propuestas o teorías desarrolladas para tal fin. Una gran parte de los estudios revisados, destacan la importancia de la creatividad en áreas como la filosofía, el arte, la administración, la política, la psicología y la educación. De esta forma, se ha aumentado el interés de los investigadores para ampliar

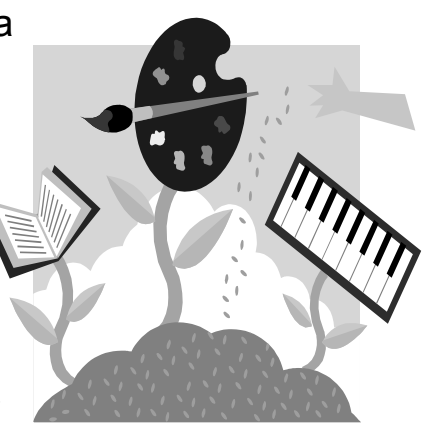
el campo de acción, facilitando un mayor acercamiento del concepto de la creatividad con aspectos como la inteligencia, personalidad, motivación y el movimiento humano.

A pesar de que es difícil llegar a un punto de entendimiento sobre el concepto y la naturaleza de la creatividad, se trata de enfocar la atención en la forma cómo se favorece el pensamiento creativo de las personas, de los procesos que se siguen y de la importancia que tiene este aspecto, principalmente, en el medio educativo y en las ciencias del movimiento humano. Por ello, el propósito de esta revisión será analizar críticamente el concepto de creatividad y tratar de integrar las diferentes teorías que aparecen sobre este tema.

\section{Definiciones de creatividad}

Para acercarse al constructo de creatividad, se debe iniciar una exploración de su definición, ya que se encuentran no solo palabras que parecen sinónimas, sino también diversas orientaciones sobre este constructo basadas en teorías antiguas y modernas. En algunos casos se le da más importancia al proceso, al potencial o las condiciones o capacidades innatas de las personas para crear. En otras situaciones, se brinda un enfoque sobre el producto creativo o se considera que debe ser una mezcla de ambas líneas de 
pensamiento, pero también se estudia la influencia que tiene el ambiente sobre las personas creativas.

Es importante tomar en cuenta los sinónimos con los que se ha relacionado la palabra creatividad. Monreal (2000) manifiesta que al iniciarse la investigación sobre la creatividad, la palabra más utilizada fue la de "genialidad". También se emplearon otros sinónimos como "originalidad", "productividad", "inventiva" y "descubrimiento", y en otros ámbitos diferentes de la psicología se le asemeja con "fantasía" e "imaginación". Para este investigador, el término creatividad mantiene un predominio importante a partir de 1950; pero afirma que no hay una unificación de este concepto. Por su parte, Corbalán, Martínez y Donolo (2003) expresan que es delicado distinguir creatividad de otros conceptos como "genialidad", "superdotación" o "arte" y que se debe tener paciencia para llegar a un consenso respecto a la definición de este complejo constructo.

Las orientaciones que enmarcan la creatividad, la definen principalmente en tres líneas de trabajo. Primero, como un proceso, luego como un producto, enfatizando en la persona creativa, y tercero, como una combinación de factores. Este planteamiento sobre el proceso y el producto creativo, es explicado por Goñi (2000), quien indica que la expresión "proceso creativo" podría ser una secuencia de pasos o etapas utilizados para resolver un problema, o que puede representar un cambio perceptual rápido o la transformación que se dispone, cuando se produce una nueva idea o solución a un problema. Sin embargo, también puede referirse a las técnicas o estrategias que utilizan las personas creativas, ya sea consciente o inconscientemente, para producir una nueva idea o combinación, relación, significado, percepción o transformación. Así, un producto creativo es un trabajo que es aceptado en cuanto a su utilidad por un grupo en algún momento. Esta aceptación se ubica en el producto y no sobre el proceso; un ejemplo de esto se presenta con las producciones artísticas que tuvieron un proceso dentro del individuo; pero que se consideran valiosas por el producto mismo hasta mucho tiempo después de su primera presentación.

Anteriormente, se habían presentado otras definiciones de creatividad y de la persona creativa. Por ejemplo, De Haan y Havighurst (1961) indican que la creatividad es cualquier actividad que lleve a la producción de algo nuevo, puede ser una invención técnica, un nuevo descubrimiento en ciencia o una nueva realización artística. Por su parte, Gardner (2001, p.126) considera que "el individuo creativo es una persona que resuelve problemas con 
regularidad, elabora productos o define cuestiones nuevas en un campo de un modo, que al principio, es considerado nuevo, pero al final llega a ser aceptado en un contexto cultural concreto". Sternberg y Lubart (1997) también se centran en la persona creativa y manifiestan que ésta se considera así, cuando genera ideas relativamente nuevas, apropiadas y de alta calidad. Sin embargo, Goñi (2000) expone que la creatividad es una forma ideal de comportamiento y se centra en la capacidad de las personas talentosas, que pueden contribuir significativamente, tanto en la sociedad como en la vida misma. Trigo y otros (1999, p. 25), afirman que "la creatividad es una capacidad humana que, en mayor o menor medida, todo el mundo posee". En esto concuerda Menchén (2001, p. 62), quien recientemente plantea que la creatividad es una característica natural y básica de la mente humana y que se encuentra potencialmente en todas las personas.

Torre, citado por Trigo y de la Piñera (2000, p. 65-66), reúne cuatro ámbitos de la creatividad enfocados en categorías de personas, que son el genio creador, con condiciones excepcionales para la creatividad; la persona creadora, que expresa su creatividad en obras de valor; la persona creativa, involucra a cualquiera que tenga el potencial para hacer cosas nuevas, mejores o diferentes, y la persona pseudo-creativa, que utiliza la creatividad para destruir o generar corrupción. Monreal (2000, p. 46) opina que este término de creatividad "se aplica tanto a las personas (creadores, creativos) como a los productos (ideas u obras creativas) o a los procesos creadores o a los entornos y ambientes (educación creadora, familia creativa) y son muchos los componentes que la integran". Para Corbalán y otros (2003, pp. 13-14), la creatividad es una integración de algunas dimensiones, según ciertas condiciones e interrelaciones. Estas dimensiones pueden ubicarse, por ejemplo, desde un plano cognitivo, socio-emocional, educativo o de dominio de destrezas, entre otros aspectos. Por su parte, Trigo y de la Piñera (2000), consideran que la creatividad tiene que ser vista como un potencial que se puede desarrollar y que debe integrarse con otras habilidades del pensamiento.

En el campo relacionado con la educación física, o a lo que actualmente se le denomina como las ciencias del movimiento humano, Rebel $(2000$, p. 88), refiriéndose a la importancia del lenguaje corporal como un elemento importante para manifestarse, indica que

la creatividad significa desarrollar la fantasía en todos los ámbitos posibles, independientemente de las consideraciones de valor y las normas, y se manifiesta por 
la tendencia a la experimentación, la inadaptación, la independencia, y por el frecuente cambio o la ocupación simultánea con varias ideas.

Torrance, entrevistado por Dunn (2000), propone tres definiciones; una en la que los individuos enfrentan una situación, en donde no se tiene una solución o no se ha aprendido. La otra es una definición artística que tiene que ver con el uso de sensaciones y de cada parte del cuerpo (muy ligada al concepto de ciencias del movimiento humano). Por último, expresa la definición de investigación que tiene relación con el proceso en donde la persona se da cuenta de que existen algunas dificultades o hay una nueva idea y hace varias pruebas hasta que obtiene la respuesta y la comunica.

En síntesis, el proceso creativo involucra todos estos aspectos. De este modo, las orientaciones que se han planteado permiten determinar la línea de trabajo que se desea seguir para estimular la creatividad, ya sea como proceso, producto o enfocándose en la persona creativa. Para ello se cuenta también con diferentes propuestas de modelos y teorías que apoyan este trabajo.

\section{Teorías sobre creatividad}

Con respecto a las teorías sobre la creatividad, se han encontrado diferentes enfoques. Las teorías que se nombran a continuación, han sido desarrolladas, en su mayoría, por investigadores interesados en este tema. Según Corbalán y otros (2003, p. 15), "existe una confluencia importante entre las teorías actuales de la creatividad que, aún desde paradigmas y métodos diversos, están haciendo propuestas perfectamente compatibles e integradoras".

Algunas teorías mencionadas por Solar (1991, p. 26-27), y Molina, Pierre y Sáenz (1995, p. 43-44), son la teoría psicoanalítica; la teoría perceptual de la Gestalt, en donde se usa el término de "pensamiento productivo" y el de "solución de problemas"; la teoría humanista, en donde los factores sociales e interpersonales cumplen un papel importante para el desarrollo o el bloqueo a la creatividad. También se menciona la teoría factorial, en donde se ubica a Guilford y Torrance, y en la que se estudia el comportamiento creador por métodos experimentales y teóricos. Finalmente, se nombra, más recientemente, la teoría neuropsicofisiológica, basada en la lateralización y codificación de los hemisferios cerebrales. 
Goñi (2000, pp. 69-105) ubica también a diferentes autores que brindan aportes significativos con sus planteamientos y teorías, entre ellas se citan: la teleología del acto creativo, en donde los procesos creativos surgen de acuerdo con ciertas exigencias en el nivel teleológico en la actividad mental. También se menciona la teoría denominada vida y creación, que se enfoca en el ser artístico; la teoría de la psicología analítica y arte poético, en donde se muestra un enfoque anti-racional de la creatividad. Por otra parte, se mencionan las teorías sobre los procesos mentales preconscientes y que continúan la línea del estudio de la regresión al servicio del ego; la teoría que da seguimiento a la labor artística llamada descubrimiento en el proceso creativo; la teoría de los enfoques psicológicos de ciencia y creatividad, que se concentra en el proceso creativo; la teoría de la psicología de la creatividad, que está muy relacionada con las diferencias individuales y se propone el estudio por medio de pruebas y escalas de medición.

Dos de las teorías en donde se han realizado investigaciones son las de Guilford y la de Torrance. Por una parte, la teoría denominada análisis de factor, intelecto y creatividad se le atribuye a Guilford. En esta se mencionan tres categorías: contenido, operación y productos. Dentro de la categoría de operación, sobresale el factor general de producción divergente que se considera una operación creativa, que está representado por la fluidez, la flexibilidad y la originalidad de los procesos de pensamiento. La otra teoría de gran influencia es la de la educación y la creatividad, del psicólogo educativo Paul Torrance, quien realizó múltiples investigaciones en relación con el pensamiento divergente y desarrolló diferentes pruebas sobre los procesos del pensamiento creativo, sobre las cualidades de los productos y de las personalidades creativas.

Existe una teoría enfocada en la creatividad e inteligencia en niños, de Wallach y Kogan (1971), quienes descubrieron variaciones al relacionar el coeficiente intelectual (CI) con las puntuaciones de pruebas de creatividad. Goñi (2001, pp. 116-124) también se refiere ampliamente a la teoría sobre el proceso creativo del psicólogo Sarnoff Mednick, llamada base asociativa del proceso creativo, que se enfoca en la naturaleza del pensamiento creativo y los elementos de asociación que realiza la persona basándose en asociaciones nuevas para llegar a la solución creativa. Por último, Goñi (2001, pp. 125-131) nombra a Carl Rogers, quien desarrolló una forma de psicoterapia centrada en el paciente y enfatizó la importancia de la unicidad del individuo, el cual cuenta con factores creativos particulares. Su teoría se denomina: hacia una teoría de la creatividad. 
Corbalán y otros (2003) hacen un resumen de los modelos; algunos nombrados también por Goñi (2000). Mencionan en primera instancia, la línea mágica o poética; el enfoque biológico, el ambiental que busca la integración entre lo hereditario y lo ambiental, las teorías que tratan la creatividad como producto de la personalidad y que está basada en rasgos y, por último, plantean el modelo que trata la creatividad como producto cognitivo; se ubica en este último enfoque a Guilford y a Sternberg.

Guilford (1971, p. 13) indica que el año de 1950 marca una etapa importante para que se mire con interés el tema de la creatividad, a partir de su discurso ante la American Psychological Association (APA). Gardner (1995, p. 38) considera que "la idea clave en la concepción psicológica de la creatividad, ha sido la de pensamiento divergente". También Gardner (1995, p. 40) cree que como investigador cognitivo, Robert Sternberg ha sido uno de los que ha descrito los modos en que los creativos identifican los problemas y la solución a los mismos $y$, entre otras cosas, la forma como estas personas reflexionan sobre sus propios procesos creativos.

En síntesis, los diferentes términos asociados a la creatividad, provienen de teorías elaboradas por investigadores reconocidos que, de una manera experimental o no, le dan seguimiento a sus hallazgos, intercambiando información o haciendo observaciones críticas a lo planteado por otros autores.

\section{Indicadores de la creatividad}

Los investigadores de la creatividad, tales como Rodríguez (1995, p. 55), Csikszentmihalyi (1998), Monreal (2000, p. 226), Corbalán y otros (2003, p.18), señalan que existen grados o niveles de creatividad para describir a las personas. Con este planteamiento, se refieren a esas condiciones que todas las personas tienen. De esta forma, Monreal (2000) es enfático al afirmar que lo que existe son diferentes grados de creatividad en los individuos. También Penagos y Aluni (2000) expresan que todas las personas son creativas, aunque sí es posible establecer niveles de creatividad y reconocer que hay personas que están excepcionalmente dotadas, tales como Freud, Gandhi o Einstein; estos dos últimos personajes estudiados por Gardner (1995) en su libro sobre las mentes creativas. 
Por su parte, Csikszentmihalyi (1998), separa a las personas creativas en varios niveles: a) los que expresan pensamientos no frecuentes: personas brillantes, interesantes y estimuladoras; b) los que experimentan el mundo de manera nueva y original: son los personalmente creativos; y c) los que realizan cambios significativos en la cultura: producen cambios en un dominio o lo transforman en otro. En cambio, Monreal (2000) indica que las formas de la creatividad son infinitas e impredecibles y tienen que ver con dos tipos de disposición: la personal y su relación con el ambiente. Se puede contar con una forma de creatividad expresiva, cuya característica es la espontaneidad y la libertad, la alegría de vivir. También se encuentra la creatividad técnica, en donde domina más la habilidad que la espontaneidad; supone una gran pericia del sujeto en creación de productos. La creatividad inventiva, donde se ubica a los inventores, cuya característica es la ingeniosidad con los materiales. Se define también al creativo innovador, quien ha sido capaz de asumir los principios elaborados por otros, pero que elabora una modificación por medio de aproximaciones alternativas y la creatividad emergente en donde se sitúa a los individuos que han tenido una influencia histórica muy relevante, ya que se conocen como genios.

Corbalán y otros (2003, p. 64), presentan en el Manual CREA una serie de criterios interpretativos generales sobre las características creativas de sujetos, basados en los percentiles y agrupados según la puntuación obtenida en la prueba respectiva como creatividad alta, media y baja. Por ejemplo, en la posición alta se indica que los sujetos tienen excelentes posibilidades para desarrollar tareas de innovación y producción creativa.

Para Penagos y Aluni (2000, p. 2), las características aceptadas de la creatividad son la fluidez, la flexibilidad, la elaboración y la originalidad. Para Rodríguez (1997), la fluidez es la cantidad de ideas que una persona puede producir respecto a un tema determinado, la flexibilidad es la variedad y heterogeneidad de las ideas producidas en donde se abordan los problemas desde diferentes ángulos y la elaboración se refiere a la factibilidad de que las ideas inventadas sean realizables. Penagos y Aluni (2000, p. 4), explican que la originalidad es la característica que define la idea, el proceso o el producto como algo único o diferente.

De manera similar, Rodríguez (1995, pp. 56-57) indica que los factores fluidez, flexibilidad y originalidad son funciones del pensamiento divergente o lateral, distinto del pensamiento convergente, lógico o vertical y expresa que el pensamiento convergente se relaciona más con el aprendizaje escolar, tal y como se desarrolla en las instituciones 
educativas, el pensamiento divergente se vincula más con la creatividad. Guilford, citado por Goñi (2000, p. 104), menciona que "los individuos muy creativos pueden generar ideas a un ritmo rápido (fluidez), romper lo establecido a fin de atacar los problemas desde una perspectiva nueva (flexibilidad) y generar ideas nuevas y genuinamente diferentes (originalidad)".

En resumen, el objetivo de contar con ciertos indicadores de la creatividad, motiva la realización de una clasificación de las personas, tomando en cuenta características entre las que sobresalen los factores de fluidez, flexibilidad y originalidad.

\section{Conceptos afines a la creatividad}

Las contribuciones de una gran cantidad de estudios con los que, desde hace muchos años se busca caracterizar y darle sentido al término de la creatividad, motivan que se despierte el interés y se continúen líneas de acción, ya sea en campos específicos o relacionando la creatividad con áreas de trabajo, como las de la personalidad, la motivación, la inteligencia, la sociedad - cultura y el género (Figura 1).

Figura 1. Conceptos relacionados con el constructo de creatividad

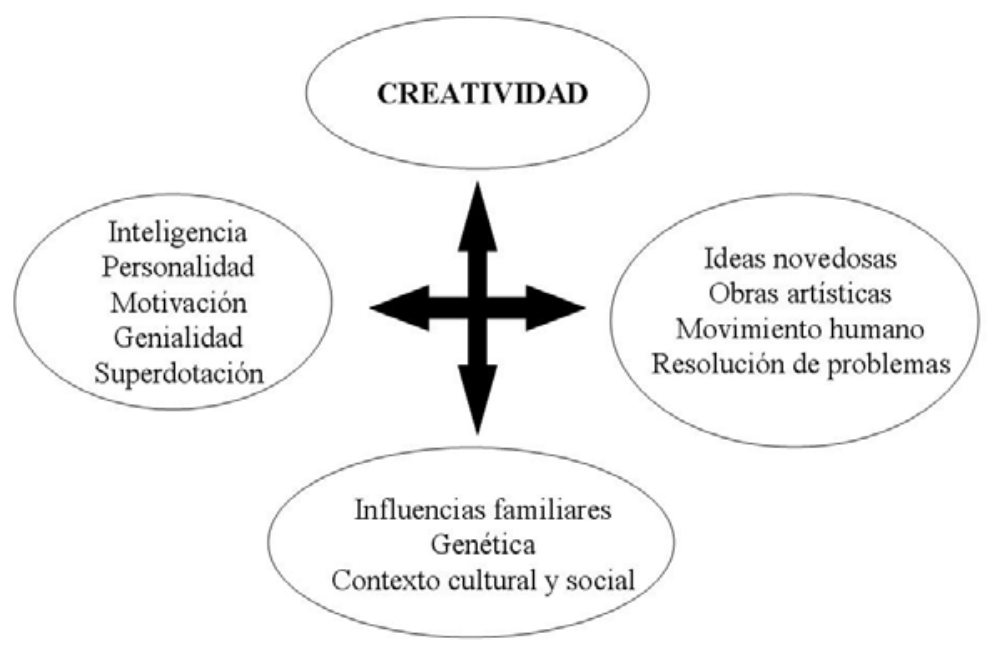




\section{Personalidad y creatividad}

Una amplia cantidad de investigadores, entre los que se encuentran Amabile (1993), Eysenck (1993), Helson (1996), Feist (1998), Wolfradt y Pretz (2001), Gelade (2002), Francis, Fearn y Brin Booker (2003), estudiaron la relación entre la personalidad y la creatividad. Por ejemplo, Feist, citado por Monreal (2000), expresa que frecuentemente, la mayoría de los psicólogos definen la personalidad en términos de diferencias individuales; es decir, en determinados rasgos que son evidentes en unas personas pero no en otras; o que se dan por igual pero con diferente intensidad, en términos de la consistencia de la conducta, en cuanto al comportamiento de las personas ante situaciones diferentes o a lo largo del tiempo.

Feist y Barron (2003), mencionan que el efecto de la personalidad sobre la creatividad es un tópico muy estudiado en los últimos 50 años, debido, en parte, al interés común por estos dos aspectos sobre las diferencias individuales. Las conclusiones principales de un meta-análisis realizado por Feist (1998), indican que en general, las personas creativas son más autónomas, introvertidas, con disposición hacia nuevas experiencias, incrédulas, seguras de sí mismas, se aceptan a sí mismas, son ambiciosas, dominantes, hostiles e impulsivas.

Monreal (2000, p. 195), define la personalidad como "todas las características psicológicas que no son cognitivas". Sin embargo, para Sternberg y Lubart (1997, p. 221), la personalidad no es una condición fija en las personas, sino que se produce un conjunto de rasgos de personalidad que son más o menos estables a lo largo de la vida. Estos rasgos son la perseverancia ante los obstáculos, la voluntad de asumir riesgos sensibles, la voluntad para crecer, tolerancia de la ambigüedad, apertura a la experiencia, fe en uno mismo, y el coraje de las convicciones propias.

Figura 2. Características de una persona creativa.

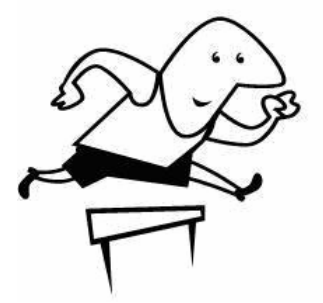

perseverancia ante obstáculos, confianza en sí misma, ambición, imaginación. 
Eysenck (1993), plantea tres tipos de variables para obtener resultados creativos. En primera instancia menciona las variables cognitivas, en donde se destacan la inteligencia, los conocimientos, las habilidades técnicas y el talento especial. Luego menciona las variables ambientales, como los factores políticos, religiosos, culturales, socio-económicos y educacionales. Por último, se refiere a las variables de personalidad, que son la motivación interna, la confianza y la disconformidad.

Por su parte, Gardner (2001, p. 129), menciona algunos rasgos de la personalidad comúnmente presentes en los creativos:

Los estudios de personas muy creativas indican que éstas tienden a destacar más por la configuración de su personalidad que por su puro poder intelectual. Cuando ya son capaces de realizar obras que se consideran creativas, difieren de sus compañeros en cuanto a ambición, confianza en sí mismos, pasión por su trabajo, insensibilidad a la crítica y por su deseo de ser creativos, de dejar huella en el mundo.

Para Maslow (2001, p. 86) la actitud creativa requiere fortaleza y coraje e indica que los estudios sobre personas creativas presentan algunas características relacionadas con esta condición como la obstinación, la independencia, la autosuficiencia, algo de arrogancia, fuerza de carácter y del ego. El miedo y la debilidad pueden alejar la creatividad o hacer que sea menos probable encontrarla. También Penagos y Aluni (2000, p. 2), consideran que la motivación, la autoestima, los estilos cognitivos, la capacidad de logro y la tolerancia a la frustración son variables de la personalidad que pueden tener relación con la creatividad.

Por su parte, Pawlak (2000, p. 33) indica que un innovador exitoso debe poseer imaginación, habilidad para trabajar en equipo, persistencia ante los obstáculos y la facilidad de percibir y medir las oportunidades. También sugiere que debe ser independiente, flexible y motivado, quizás altamente competitivo y con coraje, además puede servirle tener una carácter fuerte. Por su parte, Menchén (2001, p. 185) se refiere al perfil del profesor creativo y describe algunas características de personalidad comunes entre los creativos: son sensibles, flexibles, imaginativos, manifiestan empatía con todos los estudiantes, cuentan con recursos ingeniosos e interactúan positivamente con los demás, también les gusta arriesgarse y emprender trabajos difíciles. 
Una persona creativa tiende a mostrar un conjunto particular de cualidades de personalidad; entre ellos se encuentra un elemento importante que es la posibilidad de asumir riesgos. También se considera que el creativo necesita demostrar su valor al enfrentarse a los obstáculos (Sternberg y Lubart, 1997, p. 24). Murcia (2003, p. 30) cita un estudio en relación con este tema, realizado por Docal y Matejik en 1997, en donde se aplica un examen psicológico a padres e hijos. En los resultados, se destacan las relaciones entre el estímulo, la creatividad, los rasgos de personalidad como el carácter aventurero, empatía y miedos. Se llega a la conclusión de que los padres son más reservados en sus empeños por solucionar los problemas y se arriesgan menos que los niños.

En resumen, la personalidad de los creativos parece centrarse en un conjunto de características que se manifiestan con mayor intensidad en algunas personas. Hay rasgos que nombran algunos de estos autores y que son coincidentes entre sí, ya que recalcan la importancia de la perseverancia ante los obstáculos, la confianza en sí mismo, la ambición y la imaginación.

\section{Motivación y creatividad}

La motivación se plantea desde dos perspectivas; la intrínseca, es la que le permite al individuo tomar las decisiones con base en una fuerza interior. La extrínseca está regida por factores externos con el fin de alcanzar una meta externa como el reconocimiento, la recompensa económica o ganar una competencia (Romo, 1997, p. 154). Estas formas de la motivación se han relacionado con la creatividad y se ha estudiado la influencia tanto de una como de otra, sobre el proceso creativo.

Para Torrance, entrevistado por Dunn (2000, p.2) la personalidad y la motivación son factores que llegan a ser muy importantes en la vida de las personas; algunas pierden el interés y se detienen ante un reto creativo por falta de coraje y persistencia. Sternberg y Lubart (1997, p. 258) consideran que para ser realmente creativo, es necesario estar motivado, ya sea que existan metas extrínsecas, como el poder, el dinero o la fama; o intrínsecas como el desafío personal o la expresión de uno mismo. Los innovadores deben ser enérgicos, productivos y estar motivados por metas. 
Figura 3. Dos tipos predominantes de motivación.

Motivación intrínseca

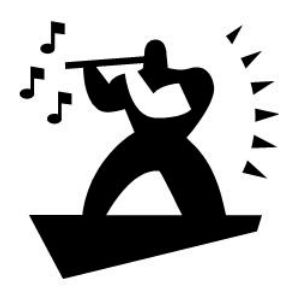

Motivación extrínseca

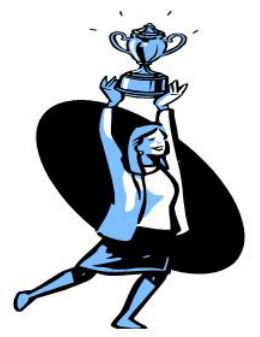

Furth y Waches (1978) creen que así como el desarrollo y el aprendizaje están interrelacionados, también los aspectos de la motivación están presentes en la adquisición de cualquier conducta. Para estos investigadores, en la enseñanza infantil se debe brindar más importancia al pensamiento, y su estructura debe enfocarse en la motivación intrínseca del niño y la niña; así en términos de comportamiento el y la infante deben ir formando sus propios esquemas de recompensas y refuerzos intrínsecos y no esperar aprobación ni premios extrínsecos de sus maestros.

Para Amabile (1996) la motivación intrínseca es una buena guía de la creatividad, pero la motivación extrínseca más bien puede perjudicarla, principalmente si es algo que se le ha impuesto a una persona. De manera similar, Sternberg y Lubart (1997, pp. 248-249) indican que la motivación es el incentivo que conduce a cierta acción; la intrínseca es considerada muy valiosa para la creatividad, ya que hace que las personas, de forma consciente e intencional, se centren en una labor que realizan, no porque se les ordena, sino porque realmente la desean hacer. Sin embargo, estos investigadores indican que la motivación extrínseca puede ser una herramienta peligrosa, ya que puede limitar la creatividad. Sternberg y Lubart (1997, pp. 250-251) citan dos estudios con alumnos universitarios; uno realizado por Amabile, en donde los sujetos escribieron poemas antes y después de pensar en razones extrínsecas para escribirlos. Estos mostraron una creatividad decreciente en los poemas escritos, atendiendo a las motivaciones externas. La otra investigación dirigida por Hill en el año de 1991, concluyó que había una correlación positiva entre el disfrute de la escritura creativa y la calidad del producto, al contrario de la motivación por obtener buenas calificaciones y reconocimiento, las cuales estaban correlacionadas negativamente con la calidad del producto. 
Lo que plantean Sternberg y Lubart (1997, p. 251), es que, "básicamente, las personas motivadas extrínsecamente, no tienen demasiado cuidado en relación con el modo como llegan a donde van, mientras puedan llegar rápido y recibir la recompensa que comporta el hecho de llegar alli". Para Amabile, citada por Sternberg y Lubart (1997, p. 254), aunque la motivación extrínseca, en ausencia de la intrínseca, puede minar la creatividad, la combinación de ambas logra intensificar la creatividad. Sternberg y Lubart (1997, p. 255) apoyan esta posición de Amabile, planteando que las personas creativas pueden hacer lo que les gusta (motivación intrínseca) y, al mismo tiempo, logran ser recompensadas (motivación extrínseca) por desarrollar su creatividad.

En resumen, diferentes investigadores plantean claramente la necesidad de incentivar más la motivación intrínseca; pero buscando también resultados positivos mediante la motivación extrínseca. La motivación intrínseca requiere de un proceso y del estímulo de esa fuerza interior para asumir con persistencia las tareas creativas. La motivación extrínseca se debe manejar con cuidado, porque podría resultar insuficiente para cumplir con las metas propuestas, así que lo que se propone es contar con una combinación que permita un buen desarrollo creativo.

\section{Inteligencia y creatividad}

Wallach y Kogan (1971, p. 72) consideran que la inteligencia es un conjunto de aptitudes que están interrelacionadas y que se refieren a la retención, transformación y utilización de los símbolos verbales y numéricos y en donde se requieren la memoria, la aptitud para resolver problemas y las destrezas de manipular y enfrentar conceptos. Estos autores realizaron estudios que indicaron que los índices de creatividad y los de inteligencia tendían a ser independientes entre sí y, mediante los datos obtenidos, confirmaron que en los escolares, la creatividad es un tipo diferente de excelencia cognitiva en comparación con la inteligencia general.

Feist y Barron (2003) también indican que la inteligencia es talento y destreza en el procesamiento de la información, resolución de problemas y razonamiento abstracto; mientras que la creatividad es una capacidad específica, no sólo para la solución de problemas, sino también para resolverlos de manera original y con determinadas adaptaciones. Para los investigadores, estos dos factores pueden estar relacionados, pero no representan lo mismo. En esto concuerda Monreal (2000, p. 161), quien plantea que la 
mayoría de investigadores están de acuerdo en afirmar que la inteligencia y la creatividad son diferentes; pero que se relacionan. También Corbalán y otros (2003, p. 24) consideran que aunque hay afirmaciones de uniformidad entre la creatividad y la inteligencia, ya que expresan planteamientos de que son dos entidades que están muy relacionadas, estas tienen su propia autonomía.

Wallach y Kogan, citados por Monreal (2000, p. 162), son investigadores que creen que sí existe diferencia entre la creatividad y la inteligencia, pues su diferente combinación en los sujetos origina cuatro grupos distintos de individuos. Primero, se menciona el grupo de creatividad alta e inteligencia alta, en el cual las personas se caracterizan por un alto nivel de autocontrol, auto confianza, libertad de expresión, extraversión y popularidad, así como un nivel de atención e interés en el trabajo escolar. Posteriormente, se menciona el grupo de creatividad alta e inteligencia baja, en el cual se encuentran las personas conflictivas, con sentimientos de inutilidad e inadecuación, cautelosas, bajas en auto confianza, y que tienen dificultad para la concentración. El tercer grupo es el de creatividad baja e inteligencia alta, que está conformado por aquellas personas que se esmeran por tener un alto rendimiento escolar y sufren si tienen fracasos académicos, y quienes además se orientan a obtener buenas notas. Se ha indicado que su grado de ansiedad es menor que el de los otros grupos y no son problemáticos. Finalmente, se menciona el grupo de creatividad baja e inteligencia baja, en el que se concentra a los sujetos desorientados, que tienen maniobras defensivas variadas y los cuales pueden producir síntomas psicosomáticos.

Gardner (1995, p. 38), a propósito de la clasificación anterior, extrae una conclusión importante a la que llegaron los psicólogos con respecto a la relación entre inteligencia y creatividad. Para Gardner (1995, p. 38), la creatividad no es lo mismo que inteligencia; un individuo puede ser mucho más creativo que inteligente o mucho más inteligente que creativo. Gardner (1995, p. 387) hace referencia a su teoría sobre las inteligencias múltiples, basado en el estudio de siete individuos, en donde cada uno sobresalía con una inteligencia humana diferente y su avance creativo suponía el uso de símbolos, imágenes y operaciones asociadas con una inteligencia concreta que actuaba en una disciplina o campo concreto. Clasifica estas inteligencias como la lógico matemática, la espacial, la artística, la social, la intra e interpersonal, la lingüística y la cinestésico corporal. 
Sternberg y Lubart (1997, p. 20) toman en cuenta otros factores más específicos y plantean que para hacer un trabajo creativo, es necesario contar con la participación de la inteligencia, desde tres ámbitos: a) la inteligencia sintética, b) la analítica, y c) la práctica. Para Torrance, en la entrevista realizada por Dunn (2000, p. 1), es muy poca la relación que puede existir entre la inteligencia y la creatividad, principalmente entre inteligencia y creatividad verbal. Esto lo demuestran las investigaciones consistentes realizadas desde hace 30 a 40 años. Cita con un ejemplo su afirmación: si a un niño de escuela se le da una prueba (test) de creatividad y otra de inteligencia, existe un $30 \%$ de varianza compartida; si se mide inteligencia, se pierde un $70 \%$ del estudio de la creatividad. Así, la creatividad puede ser mejor como predictor de logros que de inteligencia.

En síntesis, en cuanto a la influencia que ejerce la creatividad sobre la inteligencia o viceversa, hay una mayor aceptación de que estos dos factores se relacionan, los cuales son independientes se puede hacer una clasificación de los grupos basados en características específicas. La autonomía que tiene cada factor se explica en términos de que la inteligencia se basa más en ciertas aptitudes relacionadas en el razonamiento abstracto, sumado a un mayor proceso de información. Por su parte, la creatividad se trabaja mediante capacidades específicas, originalmente bajo la premisa de la resolución de problemas.

\section{Género y creatividad}

Monreal (2000, p. 130), quien cita a autores como Cox, Roe y Simonton, indica que los datos de las investigaciones confirman "una total superioridad creativa de los varones sobre las mujeres". Las razones de esta diferencia parece que no están muy claras y las que hasta el momento se han proporcionado, no son suficientes. Una de las explicaciones se centra en que las diferencias se deben a los condicionamientos biológicos y genéticos, y a que tradicionalmente se realizan más investigaciones en hombres que en mujeres.

Eysenck, citado por Monreal (2000), es un investigador del Instituto de Psiquiatría de la Universidad de Londres, el cual se basa en una teoría de creatividad desarrollada bajo el paradigma de la personalidad para explicar la aparentemente baja creatividad de las mujeres. En esta teoría se investigan dos rasgos como fuentes potenciales de las diferencias entre hombres y mujeres, a saber, el psicoticismo y el neuroticismo. Para este investigador, los hombres obtienen puntajes más altos en psicoticismo que las mujeres, y estas por su 
parte, obtienen puntajes más altos en neuroticismo en comparación con los hombres. Estas diferencias en la personalidad, según Eysenck, disponen más a los varones para la creatividad porque las características de los psicóticos se relacionan más con este constructo.

Por supuesto que el planteamiento de Eysenk ha sido criticado por otros investigadores. Para Guilford, citado por Corbalán y otros (2003, p. 22)

los creativos no son ni neuróticos ni psicóticos; los que puntúan alto en producción divergente tienden a puntuaciones un poco más bajas en tendencia neurótica o inmadurez emocional, lo cual es consistente con la observación común de que los neuróticos son menos creativos.

Lo que sí está claro, tanto para Eysenck, como para otros investigadores, es que hay también una serie de presiones de tipo social y cultural que favorece la creatividad y el rendimiento de los varones y han obstaculizado la creatividad de las mujeres (Monreal, 2000).

\section{Sociedad, cultura y creatividad}

Según Murcia (2003, p. 29), hay estudios que demuestran la influencia del medio social y cultural en los procesos de producción creativa. Pero para Monreal (2000, pp. 134-135), el estudio de este aspecto en relación con la creatividad es muy complicado metodológicamente, porque es imposible controlar la forma cómo cada sujeto ha vivido el ambiente en el que se ha desarrollado, aunque menciona estudios realizados desde una perspectiva de los ambientes globales que han aportado datos para la psicología social de la creatividad.

Sternberg y Lubart (1997, p. 266) presentan dos enfoques relacionados al entorno y a la creatividad; uno se refiere al entorno positivo en donde la creatividad se puede desarrollar mejor con un adecuado estimulo y un buen apoyo. Al contrario, con el entorno negativo la creatividad, también se puede desplegar y verse más estimulada, ya sea con entornos difíciles o incluso represivos. Según estos autores, es más grato contar con un entorno que estimula y valora la creatividad, pero un entorno altamente positivo puede ser utópico y se 
necesita, de todas formas, que una persona cuente con obstáculos en el camino de ese entorno favorable para desarrollar mejor su creatividad.

Monreal (2000, p. 136) comenta que desde el punto de vista familiar, "parece que predomina la interpretación de que un ambiente familiar creativo tiende a favorecer la creatividad de los niños y su desarrollo de adultos como tales". Tomando en cuenta a la familia como factor esencial de la sociedad, es importante que ésta se involucre en el desarrollo inicial creativo. A propósito, Murcia, Vargas y Puerta (1998, p. 65) indican:

La familia es el primer y principal agente que obstaculiza o favorece el desarrollo creativo. Dicho comportamiento lógicamente está mediado por las posibilidades culturales de la familia, que al otorgar al niño las herramientas, los signos y símbolos que conforman esa cultura, pueden estar potenciando los procesos creativos o pueden estarlos limitando, al proponer solamente los estereotipos de esa cultura.

Torrance, citado por Lagemann (1971, pp. 31-32), realizó diversos estudios en el campo educativo, en los cuales analizaba las características de personalidad utilizadas para describir a los niños creativos. Con base en esos estudios, se llegó a definir que los niños creativos aceptan el desorden; son juguetones; expresan ideas fuera de lo común; son sensibles a las emociones; son contradictorios; afrontan riesgos, y poseen mucha energía. Se determinó también la concepción que tienen los docentes sobre los rasgos de personalidad que consideran más importantes y que recompensan a menudo. En orden de preferencia se encuentran los siguientes: a) prontitud para realizar una tarea, b) cortesía, c) popularidad, d) receptividad hacia las ideas de los demás, e) aceptación de críticas, y f) buena memoria. Por el contrario, mencionaron como los rasgos menos deseables: a) ser audaz, b) siempre hace preguntas, c) atrevido, d) incapaz de aceptar la palabra de otros, e) deseoso de aceptar riesgos, y f) visionario.

Sternberg y Lubart (1997) mencionan un estudio de Chambers, realizado con 671 profesores universitarios, los cuales se escogieron por sus características de influencia, tanto positiva como negativa en la creatividad. Se encontró que los educadores que promovían la creatividad impartían sus clases informalmente, interactuando con los estudiantes y tomando en cuenta sus propuestas de trabajo. Las características principales de estos docentes, eran que trataban a sus estudiantes como personas; propiciaban la independencia y servían como modelos, para roles creativos. Por otra parte, los educadores que inhibían la creatividad, no 
tomaban en cuenta nuevas ideas, se basaban en el aprendizaje repetitivo y eran inseguros o rígidos. Monreal (2000, p. 136) refiriéndose a este mismo tema, indica que un ambiente educativo que fomente la autonomía y el aprendizaje, puede impulsar la creatividad de los niños.

También hay algunas condiciones que se pueden considerar para el desarrollo de la creatividad que son la libertad en contraposición con la presión externa o los controles excesivos y un buen ambiente de apoyo, que valore positivamente los productos de las personas. Para Sternberg y Lubard (1997), pueden darse dos posiciones con respecto a este tema, que las personas creativas se vean más estimuladas en un entorno en donde se les apoye y alimente en este campo; y el otro enfoque, en donde se plantea que la creatividad se puede dar en entornos difíciles y represivos y esta es la forma como se estimula. Cualquiera de estas dos propuestas tiene consecuencias prácticas para la formación del niño, la educación y aún para los ámbitos profesional y comercial. Mencionan algunas variables que pueden afectar o estimular la creatividad, como por ejemplo, el contexto del trabajo, las limitaciones de la labor, la evaluación, la competencia, la cooperación, clima del hogar, modelos del rol, el clima escolar, el clima organizativo y la atmósfera social.

La conclusión de estos autores con respecto al tema de la creatividad en el ámbito social, es que sería importante contar con un entorno favorable, pero también con algunos obstáculos en el camino. Se reconoce que el ambiente social, familiar y cultural es relevante para el estímulo creativo. Lo ideal es tener un entorno positivo, que debe crearse con la ayuda del principal agente educativo que es la familia. También las instituciones educativas cumplen un papel importante en la creación de ambientes de trabajo favorables, reconociendo los factores que estimulan y bloquean la creatividad.

\section{Educación y Creatividad}

La creatividad se ha investigado desde diferentes áreas de trabajo; sin embargo, el tema de la educación es uno de los que más se toma en cuenta para estudiar la influencia de la creatividad. Torrance (1977) plantea que las escuelas con visión futurista se planificarán no solo para aprender, sino también para que los estudiantes piensen; las personas deben estar preparadas para las épocas de constantes movimientos. Ese es el desafío creador que debe plantearse desde el medio educativo. Pawlak (2000, p. 32) indica que las herramientas 
más poderosas para estimular una sociedad creativa, incluyen la educación creativa, un ambiente estimulante, líderes naturales y equipos de trabajo multidisciplinarios. También considera que las naciones en avanzada deben desarrollar un ambiente estimulante para que puedan surgir las mentes creativas, por lo que se necesitan programas especiales para apoyar la educación del Siglo XXI con una necesaria transformación de la educación formal. Los programas innovadores pueden empezar desde edades tempranas; en el Jardín de Infantes, los niños pueden participar en clases creativas para estimular el pensamiento innovador. La educación puede incluir experiencias que presenten la aplicación del conocimiento multidisciplinario, aún en los niveles educativos iniciales y podrían continuar durante todo el proceso educativo. Pero también se cree que aunque es mejor desarrollar la creatividad desde pequeños, no deben existir límites con respecto a la edad para mejorarla.

Considerando que la creatividad se relaciona con la motivación y, estas a su vez, con el factor educativo, hay algunos aspectos que al respecto, mencionan Sternberg y Lubart (1997). Por ejemplo, a la hora de establecer la creatividad motivadora, se podría darle menos importancia al valor de las notas, aunque con esta acción se puede encontrar resistencia en el sector docente. Otra posibilidad sería asignarle un porcentaje alto al rubro creativo dentro de los contenidos del programa, o reconocer verbalmente el trabajo creativo, ya sea de forma individual o colectivamente, así como estimular a los estudiantes para que expongan sus trabajos en exposiciones o concursos. Por último, se podría intentar utilizar diferentes fuentes de motivación de acuerdo con el tipo de estudiantes, ya que algunos pueden responder mejor con el elogio; otros con la presentación de sus trabajos o con la valoración por medio de notas.

Los métodos de trabajo y el estilo del docente son aspectos vitales para promover la creatividad de los alumnos y se debe evaluar, tanto en el nivel de instituciones educativas (desde la educación preescolar hasta la universitaria) como en el nivel individual, el proceso que se sigue para la enseñanza. Una investigación realizada por Amador (2001) con docentes y estudiantes de segundo grado de una escuela pública costarricense, mostró como parte del análisis de los resultados que los niños y niñas que participaron en el estudio, no contaban con un ambiente tan flexible dentro del aula regular. Estos alumnos demostraron que no estaban acostumbrados a tomar decisiones, a crear y resolver problemas, lo cual dificultaba la puesta en práctica de su creatividad. 
En otra investigación relacionada con estudiantes costarricenses universitarios de Antillón y otros (1981), cuyo objetivo fue la detección de la existencia de bloqueos a la creatividad en los estudiantes de último semestre de licenciatura, de algunas de las escuelas y facultades de la Universidad de Costa Rica, mediante la aplicación de la Escala de auto evaluación de los bloqueos a la creatividad creada por Nevis, Nevis y Danzig, se mostró una uniformidad de factores bloqueados y no bloqueados independientemente de la profesión elegida y los requerimientos de ésta, así como la edad y el sexo. Uno de los factores bloqueados para la mayoría de las facultades, se reflejó en el factor llamado "renuencia a dejarse ir", que tiene que ver, entre otras cosas, con el intento de encontrar soluciones. Este concepto se relaciona con el bloqueo a la iniciativa, la flexibilidad, y el riesgo, que son características de la persona creativa. Una de las observaciones de los investigadores de este estudio, es que la universidad orienta a los alumnos en los métodos tradicionales de enseñanza que se reflejan en estos resultados; pero también señalan que la creatividad no es el único requisito para desempeñarse con éxito en la profesión.

Amador (2001) considera que la creatividad es un rasgo fundamental del ser humano que debe ser estimulado para que sea parte importante de su vida. Esto es un reto para la educación, ya que toda persona tiene derecho al acceso al conocimiento y al desarrollo de sus aptitudes, intereses, facultades e inclinaciones por medio del acto educativo. Guilford (1971, p. 22) cree que con la educación creativa se pretende formar a una persona con iniciativa, plena de recursos y confianza, preparada para enfrentar problemas personales, interpersonales o de cualquier índole. Menchén (2001, p. 14) adopta una posición similar, al afirmar que "hay que estimular a los educadores en esta dirección, tratando de que surja la creatividad en las relaciones con los alumnos, procurando el desarrollo de la capacidad creativa al máximo".

Esta capacidad creativa debe ser vivenciada por el docente, el cual debe buscar los medios para utilizar esta importante herramienta, tanto en su vida personal como profesional. Corbalán y otros $(2003$, p. 16) indican que la aplicación de la creatividad en la práctica educativa, se puede brindar en dos áreas, para identificar al niño creativo, y en la docencia, como método de trabajo para el profesor o como propuesta de objetivo del currículo. Triana (1999, pp. 15-19) plantea algunas recomendaciones para los docentes que desean utilizar una enseñanza creativa. Por ejemplo, se recomienda orientar estrategias basadas en las dificultades individuales y grupales de los alumnos; realizar actos creativos dentro de las 
clases; innovar, facilitar el surgimiento de ideas creativas y darles seguimiento; alentar a los alumnos para que jueguen con las ideas; crear espacios para explorar objetos y diferentes materiales, valorar todo esfuerzo creativo y evitar el conformismo. Además, el docente deberá tener una actitud abierta para estimular el potencial creativo, así como actualizarse en las diferentes áreas del trabajo creativo, tales como en técnicas e información bibliográfica. Torrance, citado por Goñi $(2000,109)$ concuerda con los anteriores autores, al mencionar que lo más importante es "el convencimiento de que un profesor tiene que reconocer generalmente el potencial creador en un niño o una persona joven, antes de que esté dispuesto a permitirle aprender de una manera creativa o que no es habitual".

Trigo y de la Piñera (2000, p. 73 ) toman en cuenta algunos aspectos curriculares, entre ellos la metodología que:

deberá ser participativa, motivadora de nuevas experiencias, sugerente y no impositiva o excesivamente directiva. El objetivo final es que los sujetos sean seres autónomos en la búsqueda y desarrollo de su capacidad creativa en la motricidad; y no meros ejecutantes de actividades de otros.

También Corbalán y otros $(2003$, p. 31) presentan cinco criterios educativos de la creatividad que están detallados por Ambruster y que incluyen la supresión de procedimientos de inhibición; la creación de un clima que facilite la libre producción; el fomento de la motivación para la creatividad; el uso de técnicas educativas que no marginen el pensamiento divergente y el uso de técnicas creativas en grupo que ayudan a estimular a los menos motivados.

En resumen, es vital evaluar el tipo de educación que se promueve en las instituciones y el ambiente que se genera dentro de las aulas y otros espacios de aprendizaje. Uno de los aspectos claves para la transformación hacia la propuesta creativa, es la propia vivencia docente y el conocimiento que pueda tener de los instrumentos creativos con los que puede contar. Estos insumos le servirán como elementos para identificar las características creativas de sus estudiantes y como un método de trabajo para lograr el ambiente flexible y la búsqueda de los espacios, pero principalmente, para lograr una actitud de vida creativa. 


\section{Creatividad y ciencias del movimiento humano}

A continuación, se analizan algunos elementos que sustentan los estudios enfocados en la creatividad y que están relacionados con las ciencias del movimiento humano. En primera instancia, Murcia (2003, p. 31) considera que la creatividad motriz, como campo del movimiento humano, no se ha estudiado tanto como en otros ámbitos tales como las artes, la administración o algunas áreas del campo educativo.

Murcia, Vargas y Puerta (1998, p. 77 ) asocian la creatividad motriz, no solo con el movimiento sino con la calidad del mismo, también con la variabilidad y la originalidad, buscando espacio para la imaginación y nuevas formas de crear, desarrollando estas

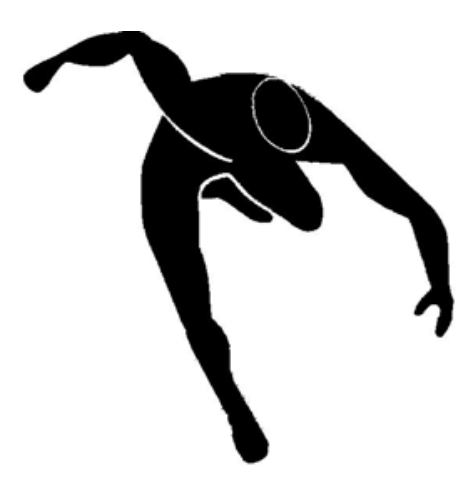
relaciones del movimiento, el pensamiento y la afectividad. Torrance, citado por Lagemann (1971) menciona que la observación del niño mientras juega puede servir como una guía para determinar su creatividad. Algunos signos que pueden ser confiables son la curiosidad que se manifiesta por medio de sus preguntas; la flexibilidad, que significa buscar otras alternativas ante un problema; la sensibilidad que tiene ante los problemas; la redefinición que se basa en la búsqueda de significados a las cosas; la conciencia de si mismo, su ser particular. También se nombra la originalidad, es decir, las ideas poco comunes, así como la capacidad de percepción que significa jugar con ideas que se presentan espontáneamente.

Murcia (2003, pp. 34-35) menciona estudios realizados en la etapa escolar en donde se determinan ambientes de juego y enseñanza; se analizan los factores de la concertación y el castigo. En algunos casos, el movimiento se castiga o se reprime, por ejemplo, al obligar a los niños a realizar ejercicios como castigo por una pérdida en un juego o actividad, o no se les permite realizar su clase de Educación Física. Otro castigo comúnmente utilizado, es no dejar a los niños salir al recreo por problemas de disciplina, desconociendo así la importancia del movimiento en la vida de los escolares, pero también considerando que los niños inmersos en ambientes negativos pueden poner a funcionar mejor su aspecto creativo, buscando soluciones inmediatas a esos obstáculos.

La educación universitaria debe ofrecer oportunidades para la educación creativa y debe procurar, por medio de sus procesos académicos y socio-culturales, la continuación de 
un proceso que permita a sus alumnos y futuros profesionales experiencias favorables para la creatividad, esto debe ser también un objetivo fundamental en el campo de las ciencias del movimiento humano. A propósito del tema, Trigo y otros (1999, p. 140-142) realizaron una investigación sobre motricidad y creatividad, con la colaboración de jóvenes estudiantes de la carrera de Ciencias de Actividad Física y el Deporte, incluyéndolos en la conformación del equipo de trabajo y participando en las diferentes etapas. Este estudio se realizó durante cinco años, al final de los cuales, los estudiantes manifestaron que su participación en este proyecto, les permitió aprender a ser más reflexivos, a tener más capacidad crítica, ser más participativos, a realizar más preguntas, a ser más flexibles, prudentes, optimistas, a autoevaluarse y a respetar opiniones de los demás.

La recomendación que brindan Trigo y otros (1999, p. 139) es que la educación universitaria debe buscar estrategias de desarrollo de los procesos cognitivos superiores que son favorecidos, a través del trabajo cooperativo de los estudiantes. Los movimientos de innovación educativa, dentro y fuera de la universidad y las investigaciones sobre los procesos de cambio social conducen a conclusiones similares. Según Bartolomé y Anguera, citados por Trigo y otros (1999, p. 139), el cambio debe originarse en el seno mismo de las instituciones y se genera por un proceso de reflexión y acción de los que componen el grupo social. Como parte de ese proceso de formación, la educación mediante el movimiento creativo, debe apoyarse en algunos indicadores tales como la fluidez, la originalidad, la flexibilidad y la elaboración. Por ejemplo, como parte de la práctica en la clase, se pueden buscar usos diferentes para un material o respuestas diversas a un estímulo rítmico; de este modo se practica la fluidez. La originalidad puede manifestarse en los alumnos al solicitarles la creación de un juego relacionado con los contenidos temáticos que están abordando en ese momento. También se puede practicar la flexibilidad, que según Trigo y de la Piñera (2000, p. 67), es la "capacidad de modificar las propias ideas en pro de un producto mejor, grupal o individual". La elaboración significa completar una idea, que se observará al realizar diferentes productos con la motricidad y las experiencias creativas, como por ejemplo, crear una historia en movimiento.

En síntesis, si las clases se realizan utilizando estilos de aprendizaje de producción y no tanto de reproducción para estimular el pensamiento divergente, se hace necesario esforzarse por plantear constantemente un ambiente apropiado para conducir a los estudiantes hacia el logro de esos objetivos motores y creativos. 


\section{Educación universitaria}

La importancia de la creatividad en la educación universitaria, es un aspecto relevante que debe ser tema de reflexión, ya que los estudiantes en esta etapa, se enfrentan a diferentes ambientes de trabajo, estrategias docentes y modelos de enseñanza. Molina, Pierre y Sáenz (1995, p. 44), plantean que dentro del marco conceptual de la Universidad de Costa Rica, "la creatividad debería ser una de las características fundamentales de nuestra institución". Sequeira, citada por Molina, Pierre y Sáenz (1995, p. 44) menciona que el enfoque curricular humanista que se debe desarrollar en la Universidad de Costa Rica, es el que más se ajusta al desarrollo de la persona creativa. Sequeira $(2000$, p. 71$)$, cita a la creatividad entre los temas que se deben clarificar en los cursos docentes universitarios, e indica "...nos hemos convertido en seres intolerantes ante el pensamiento divergente y por lo general, el docente y la docente anulan los intentos de creatividad por parte de los estudiantes y las estudiantes".

Solar (1991, p. 30) por su parte, considera que se obtienen dos beneficios recíprocos, si se educa por medio de la creatividad, los cuales ofrecen la formación de estudiantes universitarios más completos en lo humano y en lo profesional. En el proceso creativo, se presenta una posición de ruptura y de creación de patrones nuevos, logro de actitudes y aptitudes que permiten, con patrones conocidos e información nueva, reestructurar lo conocido y obtener una solución diferente. Existen diversas condiciones y situaciones que se generan en el ambiente educativo que parece, influyen en la actividad creativa de los individuos desde su infancia. Para Sequeira (2000, p. 70), el fortalecimiento de la educación preescolar, primaria, secundaria y universitaria se puede fortalecer solamente con un sistema social creativo y no de reproducción.

Mediante la educación, se debe buscar que los individuos puedan enfrentar las situaciones del presente para resolver satisfactoriamente las del futuro. Hernández (1994, p. 66) considera necesario que se puedan estimular y diferenciar los niveles de creatividad de los estudiantes para brindarles las habilidades creativas que les permitan enfrentar, con un mínimo de recursos y con mucha imaginación, el acelerado desarrollo actual. Cabe destacar, al final de este capítulo, que la creatividad es un factor que se está estudiando en el presente desde diferentes escenarios o perspectivas. Para Corbalán y otros (2003, p. 13), puede que sea difícil llegar a una conceptualización directa de la creatividad, ya que tiene 
que ver con muchos rasgos del sujeto y debe ser considerada como un constructo multidimensional en donde interactúan muchos factores.

En resumen, el sistema educativo, en el caso particular de la enseñanza superior, debe crear espacios de apertura al pensamiento divergente, de producción en lugar de reproducción, que les permita a los estudiantes y futuros profesionales, solventar los problemas de una manera creativa.

\section{Conclusión}

La creatividad, aunque difícil de definir, se estudia desde diferentes ámbitos, mediante teorías propuestas por reconocidos investigadores, las cuales sirven de apoyo para recolectar información pertinente, según las orientaciones de sus trabajos basados en la persona creativa, el proceso y el producto creativo. Se toman como referencia ciertos indicadores que son, principalmente, la fluidez, la flexibilidad y la originalidad, vinculados con la creatividad y que permiten estimular el pensamiento divergente.

En la búsqueda por encontrar un mayor sentido a este complejo constructo, se ha relacionado la creatividad con otros conceptos, en donde sobresale la personalidad. Existe el consenso de que los creativos cuentan con un conjunto de características que se manifiestan con mayor intensidad en ellos. Estos rasgos coincidentes son la perseverancia ante los obstáculos, la confianza en sí mismo, la ambición y la imaginación. También se considera importante incentivar la motivación intrínseca, pero también no se debe descuidar la motivación extrínseca, que se debe manejar con precaución, por ser un refuerzo externo. Los autores consideran que existe una relación importante entre la inteligencia y la creatividad, aunque se cree que son aspectos independientes. Se ha planteado una clasificación de los grupos basada en características específicas de las personas.

Algunos estudios se centran en la relación de la creatividad con el género y la sociedad y cultura. Sobre el primer aspecto, se llega a la conclusión de que aunque hay datos que confirman que existe una mayor creatividad en los varones que en las mujeres, se debe tomar en cuenta el ambiente social y cultural para estudiar los factores que obstaculizan la creatividad femenina; pero se hace necesario contar con más estudios para tratar este tema. Este es un amplio campo de investigación que se abre al plantear la relación del ambiente social, familiar y cultural que se considera, relevante para el estímulo 
creativo. La propuesta consiste en que se debe proveer un entorno positivo para el desarrollo del pensamiento creativo, el cual debe generarse con la ayuda del principal agente educativo que es la familia; pero también las instituciones educativas cumplen un papel importante en la creación de ambientes de trabajo favorables, reconociendo los factores que estimulan y bloquean la creatividad.

Uno de los aspectos claves para la verdadera transformación hacia la propuesta creativa, es que el docente sea un participante más de esa experiencia de aprendizaje, que vivencie la creatividad y que fortalezca su trabajo con instrumentos y métodos para estimular la creatividad de sus estudiantes, mediante un ambiente flexible que le permita proyectarse con una actitud de vida creativa.

Esta propuesta se traslada también hacia el campo de las ciencias del movimiento humano, en donde se estudia el cuerpo en acción y la relación de este con diferentes esferas del desarrollo de las personas. El significado que tiene el juego en los niños sirve como una herramienta para observar, determinar y estimular su creatividad, no para crear ambientes de juego negativos. Si se logra que los niños disfruten de un espacio agradable, podrán mostrar sus características, por medio del movimiento, asimilando la información y poniendo en práctica nuevas propuestas creativas. El desarrollo del trabajo con estudiantes universitarios, no se diferencia mucho de lo que se propone para los niños, porque las estrategias deben contar con un proceso de trabajo cooperativo, de reflexión, flexibilidad y fluidez del pensamiento y, sobre todo, de un ambiente de producción y no de reproducción.

Se hace necesario ubicar más estudios que permitan ampliar la información de lo que se está haciendo actualmente en el campo del movimiento humano, en relación con el desarrollo de la creatividad. El método de trabajo de clase utilizado por los docentes que trabajan desde nivel preescolar hasta universitario, y que están asociados a esta área, es determinante para estimular o no la creatividad. Por lo tanto, se sugiere enfocar la atención en este aspecto, orientando el estudio hacia las condiciones personales, profesionales y académicas del profesional de las ciencias del movimiento humano. 


\section{REFERENCIAS}

Amabile, T. (1993). What does a theory of creativity require?. Psychological Inquire, 4(2): $179-181$.

Amabile, T. (1996). Creativity in context. Colorado: Westview Press.

Amador, G. (2001). Cómo promover la creatividad en un grupo de niños y niñas de segundo grado de la Escuela Inglaterra. Informe de práctica dirigida para optar por el grado de Maestría en Psicopedagogía. Universidad de La Salle, Costa Rica.

Antillón, C., Castro M. I, Redondo, O., Fonseca, R., González, P., y Madrigal, M. (1981). Estudio de bloqueos a la creatividad en estudiantes de la Universidad de Costa Rica evaluados con la Escala ABC, y basado en la teoría de la Terapia Gestalt. Seminario de Graduación para optar por el grado de Licenciatura en Psicología. Universidad de Costa Rica, Costa Rica.

Csikszentmihalyi, M. (1998). Creatividad. Barcelona: Editorial Paidós.

Corbalán, J., Martínez, F., y Donolo, D. (2003). Manual Test CREA. Inteligencia creativa. Una medida cognitiva de la creatividad. Madrid: TEA Ediciones.

Dinello, R., Jiménez, C. y Motta, J. (2001). Lúdica y creatividad. Bogotá: Cooperativa Editorial Magisterio

De Haan, R. F., y Havighurst, R. J. (1961). Educating gifted children. Chicago: The University of Chicago Press.

Dunn, J. (2000). Creativity: An Interview with Dr. E. Paul Torrance. Psychology Online Journal (psychjournal.com), 1(11) November: 1-4.

Eysenck, H. (1993). Creativity and Personality: Suggestions for a theory. Psychological Inquire, 4(3): 147-178.

Feist, G. (1998). A meta-analysis of personality in scientific and artistic creativity. Personality and Social Psychology Review, 2 (4): 290-309.

Feist, G. y Barron, F. (2003). Predicting creativity from early to late adulthood: Intellect, potencial, and personality. Journal of Research in Personality, 37: 62-88.

Francis, L., Fearn, M.,y Brin B, B. (2003). Artistic creativity: Personality and the diurnal rhythm. North American Journal of Phychology, 5(1): 147-152.

Furth, H. G, y Waches, H. (1978). La teoría de Piaget en la práctica. Buenos Aires: Kapelusz.

Gardner, H. (1995). Mentes creativas. Una anatomía de la creatividad. Barcelona: Paidós. 
Gardner, H. (2001). La inteligencia reformulada. Las inteligencias múltiples en el Siglo XXI. Barcelona: Paidós.

Gelade, G. (2002). Creativity style, personality, and artistic endeavor. Genetic, Social and General Psychology Monographs, 128 (3): 213-234.

Goñi, A. (2000). Desarrollo de la creatividad. San José: EUNED.

Guilford, J. P. (1971). La Creatividad: Pasado, presente y futuro. En R.D. Strom (ed.), Creatividad y Educación (pp. 9-23). Barcelona: Ediciones Paidos.

Helson, R. (1996). In search of the creative personality. Creativity Research Journal, 9(4): 295-306.

Hernández, F. (1994). Fundamentación de un sistema de técnicas para valorar niveles de creatividad en los estudiantes. Revista Cubana de Psicología, 11(1): 65-75.

Lagemann, J. K. (1971). Procedimientos que desalientan al niño creativo. En R.D. Strom (ed.), Creatividad y Educación (pp. 24-36). Barcelona: Ediciones Paidos.

Maslow, A. H. (2001). La personalidad creadora. $7^{\text {a }}$ ed. Barcelona: Editorial Kairós.

Menchén, F. (2001). Descubrir la creatividad. Desaprender para volver a aprender. Madrid: Ediciones Pirámide.

Molina, H, Pierre, K. y Sáenz, J. (1995). El profesor universitario: Entre el conformismo y la creatividad. Revista Educación, 19(1): 41-50.

Monreal, C. (2000). Qué es la creatividad. Madrid: Editorial Biblioteca Nueva.

Murcia, N. (2003). Los condicionantes: Concertación e imposición en el desarrollo de la creatividad motriz. APUNTS, 71: 29-39.

Murcia, N., Vargas, J. y Puerta, G. (1998). El camino de la creatividad en el Educación Física y el entrenamiento deportivo infantil. Revista Educación Física y Recreación, 2(3): 59-79.

Pawlak, A. (2000). Fostering creativity in the new millennium. Research Technology Management, 43(6): 32-35.

Penagos, J. C. y Aluni, R. (2000). Preguntas más frecuentes sobre creatividad. Revista Psicología, (ed. Especial). Recuperado el 19 de julio de 2004, de http://homepage.mac.com/penagoscorzo/creatividad 2000/creatividad8.htm.

Rebel, G. (2000). El lenguaje corporal. Madrid: Editorial EDAF.

Rodríguez, M. (1995). Manual de creatividad. México: Editorial Trillas

Rodríguez, M. (1997). El pensamiento creativo integral. México: Mc Graw-Hill.

Romo, M. (1997). Psicología de la creatividad. Barcelona: Ediciones Paidós. 
Sequeira, A. (2000). Un enfoque para la Educación y la Escuela del Nuevo Siglo. Revista Educación, 24(Especial): 65-73.

Solar, M. I. (1991). Creatividad: Desafío a la función docente universitaria. PAIDEIA, 16: 2537.

Sternberg, R. J. y Lubart, T. I. (1997). La creatividad en una cultura conformista. Barcelona: Ediciones Paidós.

Torrance, P. (1977). Educación y capacidad creativa. Madrid: Ediciones Marova.

Triana, B. (1999). La alegría de crear. Bogotá: Cooperativa Editorial del Magisterio.

Trigo, E., y otros. (1999). Creatividad y motricidad. Barcelona: INDE Publicaciones.

Trigo, E. y de la Piñera, S. (2000). Manifestaciones de la motricidad. Barcelona: INDE Publicaciones.

Wallach, M. y Kogan, N. (1971). Creatividad e inteligencia en el niño. En Strom, R. D. (Ed.), Creatividad y educación. Barcelona: Ediciones Paidós.

Wolfradt, U. y Pretz, J. (2001). Individual differences in creativity: Personality, store writing and hobbies. European Journal of Personality, 15: 297-310. 